\title{
Nokardien-Pneumonie - eine Kasuistik
}

\author{
H. Frickmann ${ }^{1}$ \\ S. Jungblut ${ }^{1}$ \\ B. Böddinghaus ${ }^{2}$ \\ J. Bargon ${ }^{1}$
}

\section{Pneumonia Induced by Nocardia - A Case Report}

\section{Zusammenfassung}

Wir beschreiben den Fall einer Lungenmanifestation der Nokardiose mit Schrumpfung des rechten Oberlappens bei einem 45-jährigen Patienten ohne immunkompromittierende Vorerkrankung. Der Patient wurde in reduziertem Allgemeinzustand mit starkem Husten, rotbraunem Auswurf und Belastungsdyspnoe an uns überwiesen. Auf den Röntgenaufnahmen des Thorax waren ein dichtes Infiltrat sowie eine Schrumpfung des rechten Oberlappens zu erkennen. Nachdem im Bronchialsekret mikroskopisch und kulturell gram-positive, zum Teil verzweigte Stäbchen nachgewiesen werden konnten, wurde die Verdachtsdiagnose Nokardiose durch den Nachweise von Nocardia transvalensis mittels Polymerasekettenreaktion (PCR) bestätigt. Therapiert wurde aufgrund der Resistenzlage mit Imipenem über 5 Wochen und ab der 3. Behandlungswoche ergänzend mit Amikacin über 3 Wochen. Der Patient verließ das Krankenhaus in gutem Allgemeinzustand. Es trat kein Rezidiv auf.

\section{Abstract}

We describe a case of lung manifestation of nocardiosis with upper lobe shrinking of the right lung in a 45 year old patient without evident signs of an immuno-compromising illness. The patient came to the hospital in a reduced general state of health with severe cough, red and brown sputum and exertional dyspnoea. X-ray pictures of the thorax showed inflammatory infiltration and shrinking of the upper left lobe of the right lung. Gram-positive, branching rods were detected in the patient's bronchial secretion with the microscope and in cultures. Nocoardia transvalensis was identified via polymerase chain reaction (PCR). The antibiotic therapy was planned according to the bacterial resistance pattern. Imipenem was administered for 5 weeks and Amikacin was added for 3 weeks in the $3^{\text {rd }}$ week of therapy. The patient left the hospital in a good general state of health. There was no relapse.

\section{Einleitung}

Die Nokardiose ist eine seltene infektiologische Erkrankung, die von aeroben Aktinomyzeten des Genus Nocardia ausgelöst wird. Die Bandbreite der klinischen Manifestationen ist außerordent- lich heterogen. Sehr häufig sind pulmonale Befallsmuster anzutreffen [15]. Wir beschreiben den Fall einer Lungenmanifestation, die zu einer Schrumpfung des rechten Oberlappens des Patienten führte.

Institutsangaben

${ }^{1}$ Medizinische Klinik, St. Elisabethen Krankenhaus, Katharina Kasper Kliniken, Frankfurt/Main

${ }^{2}$ Institut für Medizinische Mikrobiologie, Klinikum der Johann-Wolfgang-Goethe-Universität,

Frankfurt/Main (V. Brade)

Anmerkung

Hagen Frickmann und Sven Jungblut sind gleichermaßen als Erstautoren anzusehen.

Korrespondenzadresse

PD Dr. Joachim Bargon · Medizinische Klinik, St. Elisabethen Krankenhaus · Ginnheimer Straße 3 .

60487 Frankfurt/Main·E-mail: bargon@em.uni-frankfurt.de

Eingang: 11. Juni $2004 \cdot$ Nach Revision akzeptiert: 15. September 2004

Bibliografie

Pneumologie 2004; 58: 850-853 @ Georg Thieme Verlag KG Stuttgart · New York

DOI $10.1055 / \mathrm{s}-2004-830099$

ISSN 0934-8387 
Der Patient (45 Jahre, 1,93 m, $84 \mathrm{~kg}$ ) wurde aus stationärer Behandlung mit therapieresistenter Pneumonie übernommen. Vorausgegangene antimikrobielle Therapien mit Penicillin, Cefuroxim, Ceftriaxon, Gentamycin, Piperacillin + Tazobactam und Clarithromycin hatten sich als nicht erfolgreich erwiesen. Er klagte über rezidivierende Fieberschübe bis $40 \mathrm{C}$, starken Husten mit rotbraunem Auswurf sowie einen Gewichtsverlust von $10 \mathrm{~kg}$ in 6 Wochen von ursprünglich $97 \mathrm{~kg}$. Allergien waren nicht bekannt, jedoch ein Nikotinabusus von 10 Zigaretten/Tag, keine Haustiere, keine Auslandsaufenthalte. Eine bekannte arterielle Hypertonie wurde mit Metoprololtatrat $50 \mathrm{mg} 1 / 2$ Tablette/Tag behandelt.

Der Patient wurde in deutlich reduziertem Allgemeinzustand mit Belastungsdyspnoe und Blässe aufgenommen. Über dem rechten Lungenfeld waren grobblasige Rasselgeräusche zu hören. Das Abdomen sowie eine grob orientierende neurologische Untersuchung waren unauffällig.

\section{Pathologische Laborwerte}

CRP 298,4 MG/L, Leukozyten 33,1 G/L (83\% Neutrophile, 9,5\% Lymphozyten), Kreatinin 1,83 MG/DL, Hämoglobin 88 g/L, Hämatokrit 0,25, Erythrozyten 3,3 T/L, MCH 3,3 pg, MCV 75,7 fL, Thrombozyten 704 G/L, BSG 115/125, Transferrin erniedrigt auf $1,4 \mathrm{~g} / \mathrm{l}$, Ferritin erhöht auf $1047 \mu \mathrm{g} / \mathrm{l}$, HIV negativ, ANCA negativ. Für ANA fand sich mit 1:320 ein positiver Befund, jedoch brachte die ANA-Differenzierung im Dot-Blot nur negative Ergebnisse. In der Lymphozytendifferenzierung waren die Konzentrationen der Gesamtlymphozyten, der CD4- und CD8-Zellen sowie der B-Lymphozyten und NK-Zellen erniedrigt.

\section{Lungenfunktion}

Bei einer guten Blutgasanalyse bestanden lediglich eine leichte restriktive Ventilationsstörung und kein Anhalt für eine Obstruktion. VC IN: 4,681 (79,9\%), FVC: 4,45 l (79,4\%), FEV 1 :3,631 (80,7\%), FEV 1\% VC IN: 77,63\%, RV: 2 l (87,2\%), RV\% TLC: 29,88\%, BGA: $\mathrm{pO}_{2}=77,93 \mathrm{~mm} \mathrm{Hg}, \mathrm{pCO}_{2}=38,03 \mathrm{~mm} \mathrm{Hg}, \mathrm{pH}=7,4$.

\section{Bronchoskopie}

Es zeigte sich ein blasses, geschwollenes und vergröbertes Schleimhautrelief im rechten Oberlappen. Reichlich zähes, grünliches Sekret lief aus den einsehbaren Ostien nach Absaugung beständig nach. Bei einer Folgeuntersuchung war eine Längsstreifung der Bronchialschleimhaut im Bereich des rechten Oberlappens zu sehen.

\section{Histologie}

In der transbronchialen Biopsie aus dem Bereich des rechten Oberlappens zeigte sich eine entzündliche Infiltration mit Basalmembranverdickung, Schleimhautödem und Schleimhautfibrose. Malignität, säurefeste Stäbchen in der Ziehl-Neelsenfärbung und Pilze waren nicht im Biopsat nachweisbar. In der Färbung nach Grocott zeigten sich jedoch im Bronchialsekret teils verzweigte Fadenbakterien, die morphologisch Nokardien entsprachen. Das Sekret enthielt reichlich neutrophile Granulozyten und einige Alveolarmakrophagen.
Tab. 1 Antibiogramm potenziell wirksamer Antibiotika

\begin{tabular}{|llcl}
\hline Antibiotikum & $\begin{array}{l}\text { Minimale Hemm- } \\
\text { konzentration } \mathbf{M H K} \\
\text { in } \mathbf{m g / l}\end{array}$ & $\begin{array}{l}\text { Grenze } \\
\text { in } \mathbf{~} \mathbf{m g} / \mathbf{l}\end{array}$ & Ergebnis \\
\hline Ceftriaxon & 0,5 & 64 & sensibel \\
\hline Imipenem & $0,02-0,19$ & 16 & sensibel \\
\hline Amikacin & $0,03-3$ & 16 & sensibel \\
\hline Ciprofloxacin & 0,5 & 4 & sensibel \\
\hline Doxycyclin & 3 & 16 & sensibel \\
\hline Cotrimoxazol & $0,03-0,25$ & 128 & sensibel \\
\hline Erythromycin & $0,03->256$ & 8 & fraglich \\
\hline
\end{tabular}

\section{Mikrobiologie}

Im Bronchialsekret sah man gram-positive verzweigte Stäbchen, die auf eine Nokardiose hinwiesen, $\alpha$-hämolysierende Streptokokken und Neisserien ohne pathologische Bedeutung sowie Imipenem- und Amikacin-sensiblen Staphylococcus aureus nach Anreicherung. Ferner wurde Candida albicans detektiert.

\section{Molekularbiologie und Antibiogramm}

Die Polymerasekettenreaktion (PCR) und Sequenzierung der bakteriellen 16S-rDNA nach Anzucht der Keime auf Middlebrook-Agar ergab Nocardia transvalensis bzw. nah verwandte Erreger. Die PCR auf M. tuberculosis und verwandte Mykobakterien war negativ. Dem Antibiogramm zufolge bestand eine hinreichende Sensibilität gegen Imipenem und Amikacin. Resistenzen gab es gegen sämtliche Penicilline, die meisten Cephalosporine sowie Gentamycin und Fusidinsäure. Das genaue Antibiogramm der potenziell wirksamen Antibiotika findet sich in Tab. 1.

Aufgrund des Mangels an Richtlinien für die In-vitro-Empfindlichkeitstestung von Nokardien wurde der gesamte Messbereich neben einer Einteilung in sensibel/resistent nach allgemeinen Grenzwerten vom Labor angegeben. In vivo sei nach Laborangaben die stärkste Wirkung durch die Kombination von Imipenem und Amikacin zu erwarten.

\section{Röntgenaufnahmen des Thorax}

Die Aufnahmen zeigten bei Übernahme des Patienten eine abszedierende Oberlappenpneumonie mit einer runden Verdichtung im apikalen Unterlappensegment. Im Verlauf kam es zu einer zunehmenden Schrumpfung des rechten Oberlappens, am ehesten als Folge einer zunehmenden Fibrosierung, mit einem verbreiterten Pleuraspalt rechts apikal. Auch eine leichte Mediastinalverschiebung nach rechts war erkennbar (Abb.1). Bei Entlassung waren diese Veränderungen des Röntgenbildes deutlich rückläufig.

\section{CT Thorax/Abdomen/Schädel}

Ein sehr dicht infiltrierter, schrumpfender rechter Oberlappen mit Spuren der Karnifizierung war auf den Thoraxaufnahmen zu erkennen (Abb. 2). Im Abdomen sah man eine grenzwertig vergrößerte Milz sowie ein thrombosiertes infrarenales Aortenaneurysma mit Lumeneinengung um ein Drittel. Im CT des Schädels (CCT) konnten intrakranielle Abszesse ausgeschlossen werden. 


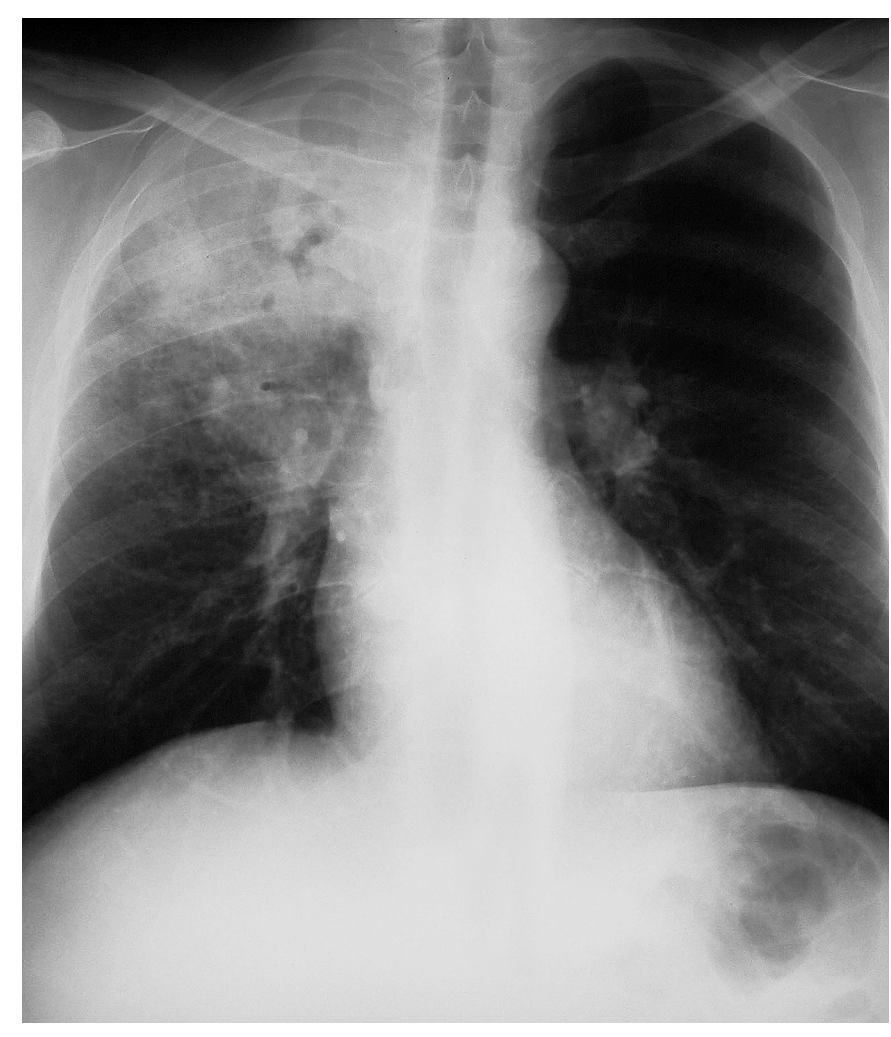

Abb. 1 Röntgenbefund des Thorax in der posterior-anterior-Aufnahme: Dichtes Infiltrat im rechten Oberlappen mit streifigen Ausläufern zum rechten Hilus, Verdacht auf mediastinale Lymphknotenvergrößerungen rechts.

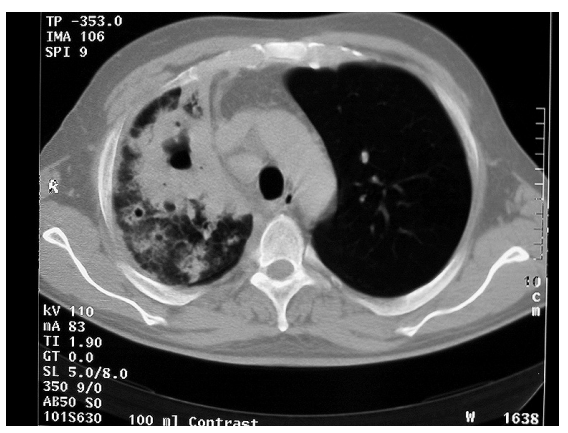

Abb. 2 CT-Thorax: Dichtes Infiltrat mit zentraler Kaverne (ohne Flüssigkeitsspiegel) im rechten Oberlappen, narbige Veränderungen subpleural sowie Pleuraverdickung, Minderbelüftung des rechten Oberlappens, deutliche Verschiebung des Mediastinums nach rechts.

\section{Echokardiographie}

Eine geringgradige Trikuspidalinsuffizienz sowie ein geringfügiger Perikarderguss bei guter systolischer und diastolischer Funktion des linken Ventrikels ohne regionale Kontraktionsausfälle wurden diagnostiziert.

\section{Sonographie Abdomen}

Eine Aortenektasie und eine schwere Aortensklerose waren darstellbar.

\section{Therapie und Verlauf}

Der Patient wurde während des fünfwöchigen Aufenthalts mit Imipenem $(3 \times 1 \mathrm{~g}$ täglich $)$ und aufgrund des Antibiogramms während der letzten 3 Wochen ergänzend mit Amikacin $(1 \times 1 \mathrm{~g}$ täglich) behandelt. Er konnte in gutem Allgemein- und Gesundheitszustand bei rückläufigen Entzündungsparametern sowie
Normalisierung des Blutbilds und des Eisenstoffwechsels entlassen werden. Bis heute kam es zu keinem Rezidiv.

\section{Diskussion}

Bei Nokardien handelt es sich um aerobe, gram-positive, verzweigte, säurefeste Aktinomyzeten. Insbesondere N. asteroides, N. brasiliensis, N. farcinia, N. nova, N. otitidiscaviarum (caviae) und N. transvalensis sind als humanpathogen bekannt. Der Erreger existiert weltweit im Boden, im Wasser und an Staubpartikel gebunden in der Luft [14]. Aufgrund zahlreicher Resistenzmuster gibt es derzeit keine antimikrobielle Standardtherapie. Jedoch werden Sulfonamide, insbesondere Trimethoprim-Sulfamethoxazol, häufig mit Erfolg verabreicht. Bei mangelnder Wirksamkeit der antibiotischen Therapie und einem fortbestehenden infektiösen Herd kann eine chirurgische Intervention notwendig werden [16].

Obwohl in seltenen Fällen auch immunkompetente Patienten an Nokardiose erkranken können, sind insbesondere immunsupprimierte Patienten betroffen. Dabei stehen Transplantationspatienten und HIV-infizierte Patienten als Risikogruppe im Vordergrund [14]. Die transplantationsassoziierte Nokardiose ist jedoch seltener als früher angenommen [12].

Neben der Immunsuppression zählen die Corticosteroidtherapie und ergänzende Lungenkrankheiten wie zum Beispiel Alveolarproteinose und Tuberkulose zu den Risikofaktoren $[8,14]$. Steroide stellen sogar einen eigenen Risikofaktor dar, da immunsuppressive Therapien unter Aussparung von Steroiden mit deutlich reduzierten Nokardioseraten einhergehen. Krankheiten wie Asthma, chronisch obstruktive Lungenkrankheit oder Autoimmunkrankheiten, die eine dauerhafte Einnahme von Steroiden nötig machen, sind daher mit einem erhöhten Nokardioserisiko verbunden [14]. Männer sind häufiger betroffen als Frauen, was möglicherweise darauf zurückzuführen ist, dass Östradiol eine gewisse Schutzfunktion gegen Aktinomyzeten aufzuweisen scheint [14]. Weitere Risikofaktoren, die durch Beeinträchtigung der immunologischen Funktion das Nokardioserisiko erhöhen, sind hämatologische oder sonstige maligne Neubildungen, Knochenmarkstransplantationen, chronisch granulomatöse Erkrankungen, Diabetes und Alkoholismus [14].

Eine Lungenbeteiligung im Rahmen einer Nokardiose findet sich am häufigsten. Es kommt jedoch gelegentlich auch zu extrapulmonalen Manifestationen. Gehirnabszesse sind die typische Komplikation einer Streuung von Nokardien, die meist von einem Lungenherd ausgeht [2].

Die kutane Nokardiose ist in vier Ausbreitungsmuster untergliedert: Die primär kutane Erkrankung, die lymphokutane Nokardiose, die Hautbeteiligung ausgehend von einem disseminierten Focus und das Mycetom [12]. In der Literatur finden sich Berichte über multiple subkutane Abszesse [5] und kutane Abszesse an Rücken, Kopfhaut und Lippen [3].

Die Nokardiose kann auch mit anderen Krankheitsbildern assoziiert auftreten, was die Differenzialdiagnose erschweren kann. Es gibt Berichte über Lungennokardiosen mit Komplikationen 
wie bei Aspergillusbefall [7] und Lipoidpneumonien [1]. Ferner wird über Nokardiose-Patienten mit Eosinophilie [11] und systemischem Lupus erythematodes [10] berichtet. In der Literatur findet sich sogar ein Fall einer mit einem Cushing Syndrom assoziierten pulmonalen Nokardiose [4].

Der Lungenbefall muss als die typische Manifestation der Nokardiose angesehen werden. In der Literatur sind multiple bilaterale pulmonale noduläre Läsionen im Röntgenbild beschrieben, die den Ausschluss von Lungenmetastasen notwendig machen können [13]. Der Lungenbefall kann mit Atelektasen [9] und einem Hydropneumothorax [6] einhergehen.

Bei der Lungenmanifestation können Fieber, Nachtschweiß, Leistungsabfall und Gewichtsverlust auftreten. Weitere typische Beschwerden sind Dyspnoe, Husten, Hämoptysen und pleuritischer Schmerz.

Ausgehend von der Lunge kann es zur Ausbreitung der Infektion kommen. Dabei sind Komplikationen wie Empyem, Mediastinitis, Perikarditis und Vena-cava-Syndrom möglich, was dann eine chirurgische Intervention nötig macht. Ein auf die Lunge lokalisierter Befall kann jedoch mit gutem Erfolg antimikrobiell therapiert werden [15].

Aufgrund der Vielfalt der klinischen Manifestationsmöglichkeiten der Nokardiose ist zur Diagnose die Kultur und mikrobiologische Identifikation der Keime nötig.

In unserem Fall hatten wir es mit einem pulmonalen Befallsmuster zu tun. Eine zerebrale Abszedierung konnte im CCT ausgeschlossen werden, Hautläsionen fanden sich nicht.

Diagnostisch wegweisend waren der histologische und mikrobiologische Befund gram-positiver verzweigter Stäbchen bei therapieresistenter Pneumonie. Das Vorhandensein lediglich unspezifischer antinuklearer Antikörper in geringer Konzentration machte einen autoimmunologischen Prozess eher unwahrscheinlich. Die molekularbiologische Detektion der NokardienDNA mittels PCR rundete die Diagnostik ab.

Die Schrumpfung des rechten Oberlappens, die eindrucksvoll im Röntgenbild zu sehen war, ist auf einen fibrotisch karnifizierenden Umbau zurückzuführen. Die rundliche Verdichtung, die zeitweise im apikalen Unterlappensegment beobachtet wurde, ist im Rahmen der Abszedierung ein möglicher Befund. Solche Rundherde, die den Ausschluss von Metastasen notwendig machen können, werden als „cannonballs“ bezeichnet [13]. Sie repräsentieren eine der vielfältigen radiologischen Befundkonstellationen der Lungennokardiose [15]. Vermutlich hängt das Phänomen der Rundherdbildung mit der Reaktion der körpereigenen Abwehr auf die Keime zusammen, wobei die lokale Ausbreitung durch neutrophile Granulozyten und Makrophagen so lange inhibiert wird, bis eine durch T-Lymphozyten vermittelte Zytotoxizität eingeleitet werden kann [14].

Die Therapie erfolgte als gezielt hochdosierte antimikrobielle Therapie mit Imipenem und Amikacin anhand des Antibio- gramms aus der mikrobiologischen Untersuchung. Die Abweichung von der Standardtherapie wurde gewählt, da die angewandte Kombination in Anbetracht der Resistenzlage nach Einschätzung des Labors in vivo die besten therapeutischen Erfolge versprach.

Nach erfolgreicher antimikrobieller Therapie konnte der Patient das Krankenhaus in guter körperlicher Verfassung ohne weitere Antibiotikagabe verlassen. Ein Rezidiv trat nicht auf.

Obwohl die Lungennokardiose ein sehr seltenes Krankheitsbild darstellt, sollte man sie als mögliche Differenzialdiagnose insbesondere im Falle therapieresistenter Pneumonien, die mit einer weiteren immunkompromittierenden Pathologie einhergehen, im Hinterkopf behalten. Da die Symptome sehr vielfältig sein können und eine Diagnose letztlich nur durch die Erregerbestimmung möglich ist, ist die Nokardiose eine schwierig zu diagnostizierende Erkrankung [14].

\section{Literatur}

${ }^{1}$ Abe M, Kondo K, Fujino S et al. Lipoid pneumonia combined with pulmonary nocardiosis caused by inhalation of amphotericin-B after renal transplantation. Nihon Kyobu Shikkan Gakkai Zasshi 1996; 34 (6): $737-740$

2 Chen YM, Yeh PF, Hwang MS et al. Pulmonary nocardiosis with cerebral abscess successfully treated by medication alone - a case report. Zhonghua Yi Xue Za Zhi (Taipei) 1991; 47 (4): 294 - 298

${ }^{3}$ Dekeyser S, Corroyer-Simovic B, Cashia M et al. Nocardia otitidiscaviarum, cutaneous infection in a patient receiving long-term corticosteroid treatment. Ann Biol Clin 2003; 61 (2): 219-222

${ }^{4}$ Dohchin A, Sato M, Yamanaka H et al. Pulmonary nocardiosis associated with Cushing's syndrom. Nihon Kokyuki Gakkai Zasshi 1999; 37 (2): $125-129$

${ }^{5}$ Enomoto M, Yamasawa H, Sawai T et al. Pulmonary nocardiosis with bilateral diffuse granular lung shadows in a patient with subcutaneous panniculitic T-cell lymphoma. Intern Med 2002; 41 (11): 986-989

${ }^{6}$ Gowrinath K, Das S, Ranjitham M et al. Nocardial hydropneumothorax. Indian J Chest Dis Allied Sci 2004; 46 (1): 51 - 53

${ }^{7}$ Holt RI, Kwan JT, Sefton AM et al. Successful treatment of concomitant pulmonary nocardiosis and aspergillosis in an immunocompromised renal patient. Eur J Clin Microbiol Infect Dis 1993; 12 (2): 110-112

${ }^{8} \mathrm{Hui} \mathrm{CH}$, Au VW, Rowland K et al. Pulmonary nocardiosis re-visited: experience of 35 patients diagnosis. Respir Med 2003; 97 (6): 709-717

9 Kuroki S, Yamada H, Katoh O et al. A case of pulmonary nocardiosis associated with middle lobus atelectasis. Kansenshogaku Zasshi 1990; 64 (2): $243-248$

${ }^{10}$ Leong KP, Tee NW, Yap WM et al. Nocardiosis in patients with systemic lupus erythematosus. The Singapore Lupus Study Group. J Rheumatol 2001; 28 (1): 215-217

${ }^{11}$ Nakagawa Y, Fukushima Y, Sakata T. Chronic pulmonary nocardiosis with eosinophilia in an immunocompetent host. Nihon Kyobu Shikkan Gakkai Zasshi 1996; 34 (8): 916 -920

12 Peraira JR, Segovia J, Fuentes R et al. Pulmonary nocardiosis in heart transplant recipients: treatment and outcome. Transplant Proc 2003; 35 (5): 2006-2008

13 Pifarre R, Teixido B, Vila M et al. Pulmonary nocardiosis as a cause of radiographic imaging multiple pulmonary nodules. Arch Bronchopneumol 2001; 37 (11): $511-512$

${ }^{14}$ Whitehurst A, Chapman SW. Basic biology and epidemiology of nocardiosis. UpToDate, 2004

${ }^{15}$ Whitehurst A, Chapman SW. Clinical manifestations of nocardiosis. UpToDate, 2004

${ }^{16}$ Whitehurst A, Chapman SW. Diagnosis and treatment of nocardiosis. UpToDate, 2004 